\title{
Upwind Schemes for the Navier-Stokes Equations
}

\author{
Barry Koren \\ Centre for Mathematics and Computer Science \\ P.O. Box 4079, 1009 AB Amsterdam, The Netherlands
}

SUMMARY

\begin{abstract}
A discretization method is presented for the full, steady, compressible Navier-Stokes equations. The method makes use of quadrilateral finite volumes and consists of an upwind discretization of the convective part and a central discretization of the diffusive part. In the present paper, the emphasis lies on the discretization of the convective part.

The applied solution method directly solves the steady equations by means of a Newton method, which requires the discretization to be continuously differentiable. For two upwind schemes which satisfy this requirement (Osher's and van Leer's scheme), results of a quantitative error analysis are presented. Osher's scheme appears to be more and more accurate than van Leer's scheme with increasing Reynolds number. A suitable higher-order accurate discretization of convection is chosen. Based on this higher-order scheme, a new limiter is constructed. Further, for van Leer's scheme, a solid wall - boundary condition treatment is proposed, which ensures a continuous transition from the Navier-Stokes flow regime to the Euler flow regime.

Numerical results are presented for a subsonic flat plate flow and a supersonic flat plate flow with oblique shock wave - boundary layer interaction. The results obtained agree with the predictions made.

Useful properties of the discretization method are that it allows an easy check of false diffusion and that it needs no tuning of parameters.
\end{abstract}

1980 Mathematics Subject Classification: 65N05, 65N30, 76N05, 76N10.

Key Words and Phrases: upwind schemes, Navier-Stokes equations

Note: This work was supported by the European Space Agency (ESA), via Avions Marcel Dassault Bréguet Aviation (AMD-BA).

\section{INTRODUCTION}

1.I. Navier-Stokes equations

The equations considered are the full, steady, 2D, compressible Navier-Stokes equations

$$
\frac{\partial f(q)}{\partial x}+\frac{\partial g(q)}{\partial y}-\frac{1}{R e}\left\{\frac{\partial r(q)}{\partial x}+\frac{\partial s(q)}{\partial y}\right\}=0
$$

with $f(q)$ and $g(q)$ the convective flux vectors, Re the Reynolds number, and $r(q)$ and $s(q)$ the diffusive flux vectors. As state vector we consider the conservative vector $q=(\rho, \rho u, \rho v, \rho e)^{T}$, with for the total energy $e$ the perfect gas relation $e=p /(\rho(\gamma-1))+1 / 2\left(u^{2}+v^{2}\right)$. The primitive flow quantities used are density $\rho$, pressure $p$, and the velocity components $u$ and $v$. The ratio of specific heats $\gamma$ is assumed to be constant. The convective flux vectors are defined by

$$
f(q)=\left(\begin{array}{l}
\rho u \\
\rho u^{2}+p \\
\rho u v \\
\rho u(e+p / \rho)
\end{array}\right), \quad g(q)=\left[\begin{array}{l}
\rho v \\
\rho v u \\
\rho v^{2}+p \\
\rho v(e+p / \rho)
\end{array}\right),
$$

and the diffusive flux vectors by

$$
r(q)=\left(\begin{array}{l}
0 \\
\tau_{x x} \\
\tau_{x y} \\
\tau_{x x} u+\tau_{x y} \nu+\frac{1}{\gamma-1} \frac{1}{\operatorname{Pr}} \frac{\partial\left(c^{2}\right)}{\partial x}
\end{array}\right), s(q)=\left(\begin{array}{l}
0 \\
\tau_{x y} \\
\tau_{y y} \\
\tau_{y y} \nu+\tau_{x y} u+\frac{1}{\gamma-1} \frac{1}{\operatorname{Pr}} \frac{\partial\left(c^{2}\right)}{\partial y}
\end{array}\right),
$$

with $\operatorname{Pr}$ the Prandtl number, $c$ the speed of sound (for a perfect gas: $c=\sqrt{\gamma p / \rho}$ ) and with $\tau_{x x}, \tau_{x y}$ and $\tau_{y y}$ the viscous stresses. Assuming the diffusion coefficients to be constant and Stokes' hypothesis to hold, the viscous stresses are 


$$
\begin{aligned}
\tau_{x x} & =\frac{4}{3} \frac{\partial u}{\partial x}-\frac{2}{3} \frac{\partial v}{\partial y}, \\
\tau_{x y} & =\frac{\partial u}{\partial y}+\frac{\partial v}{\partial x}, \\
\tau_{y y} & =\frac{4}{3} \frac{\partial v}{\partial y}-\frac{2}{3} \frac{\partial u}{\partial x} .
\end{aligned}
$$

Here, we present a discretization method which allows an accurate (and efficient) computation of (steady) high-Reynolds number flows up to and including the Euler flow regime. The challenge in developing such a method is to find a discretization of the convective part which is accurate not only for typical Euler flows, but also for typical Navier-Stokes flows, like boundary layer flows. Finding a discretization for the diffusive part which satisfies the same requirements, is thought to be easy.

\subsection{Discretization method}

To still allow Euler flow solutions with discontinuities, the equations are discretized in integral form. A straightforward and simple discretization of the integral form is obtained by subdividing the integration region $\Omega$ into quadrilateral finite volumes $\Omega_{i, j}$ and by requiring that the conservation laws hold for each finite volume separately:

$$
\underset{\partial \Omega_{i, j}}{\phi}\left(f(q) n_{x}+g(q) n_{y}\right) d s-\frac{1}{R e} \underset{\partial \Omega_{i, j}}{\phi}\left(r(q) n_{x}+s(q) n_{y}\right) d s=0, \forall i, j
$$

This discretization requires an evaluation of convective and diffusive fluxes at each volume wall.

1.2.1. Evaluation of convective fluxes. Based on experience with the Euler equations (see [5] for an overview), for the evaluation of the convective fluxes we prefer an upwind approach, following the Godunov principle [2]. So, along each finite volume wall, the convective flux is assumed to be constant and to be determined by a uniformly constant left and right state only. For the 1D Riemann problem thus obtained, an approximate Riemann solver is applied. The choice of the left and right state, to be used as input for the approximate Riemann solver, determines the accuracy of the convective discretization. First-order accuracy is simply obtained by taking the left and right state equal to that in the corresponding adjacent volume [6]. Higher-order accuracy is obtained by applying lowdegree piecewise polynomial state interpolation (MUSCL-approach), using two or three adjacent volume states for the left and right state separately [4]. For this flux evaluation, we make use of the rotational invariance of the Navier-Stokes equations in order to reduce the number of these evaluations per finite volume wall from two to one. A more detailed discussion of the discretization of the convective part is given in section 2 .

1.2.2. Evaluation of diffusive fluxes. For the evaluation of the diffusive fluxes, it is necessary to compute $\nabla u, \nabla v$ and $\nabla c^{2}$ at each volume wall. To compute for instance $(\nabla u)_{i+1 / 2, j}$, where $i+1 / 2$ refers to the volume wall separating $\Omega_{i, j}$ and $\Omega_{i+1, j}$, we use Gauss' theorem

$$
\nabla u_{i+1 / 2, j}=\frac{1}{A_{i+1 / 2, j}} \partial \Omega_{i+1, j} u \mathbf{n} d s,
$$

with $\partial \Omega_{i+1 /, j}$ the boundary and $A_{i+1 /, j}$ the area of a shifted quadrilateral finite volume $\Omega_{i+1 / 2, j}$ which vertices $\mathrm{z}=(x, y)^{T}$ are defined by

$$
\mathbf{z}_{i, j \pm 1 / 2}=\frac{1}{2}\left(\mathbf{z}_{i-1 / 2, j \pm 1 / 2}+\mathbf{z}_{i+1 / 2, j \pm 1 / 2}\right)
$$

and a similar expression for $\mathbf{z}_{i+1, j \pm 1 / .}$. The line integral in (1.6) is approximated by

with for $u_{i+1 / 2, j \pm 1 / 2}$ the central expression

$$
\begin{aligned}
& \underset{\partial \Omega_{i+h, j}}{\oint} u \text { nds }=u_{i+1, j} \quad\left(\mathbf{z}_{i+1, j+1 / 2}-\mathbf{z}_{i+1, j-1 / 2}\right)+ \\
& u_{i+1 / 2, j+1 / 2}\left(\mathbf{z}_{i, j+1 / 2}-\mathbf{z}_{i+1, j+1 / 2}\right)+ \\
& u_{i, j} \quad\left(\mathbf{z}_{i, j-1 / 2}-\mathbf{z}_{i, j+1 / 2}\right)+ \\
& u_{i+1 / 2, j-1 / 2}\left(\mathbf{z}_{i+1, j-1 / 2}-\mathbf{z}_{i, j-1 / 2}\right) \text {, }
\end{aligned}
$$

$$
u_{i+1 / 2, j \pm 1 / 2}=\frac{1}{4}\left(u_{i, j}+u_{i, j \pm 1}+u_{i+1, j}+u_{i+1, j \pm 1}\right) \text {. }
$$

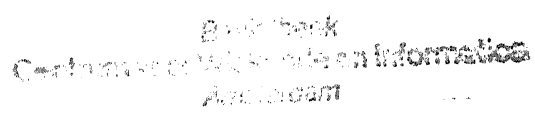


Similar expressions are used for the other gradients and other walls. For sufficiently smooth grids this central diffusive flux computation is second-order accurate. Notice that by using central expressions, as (1.9), the directional dependence coming from the cross derivative terms is neglected. No significant gain in solution quality is expected from a biased approach as proposed in [1]. Given the fact that the present diffusive flux evaluation is rather cheap, here, use of rotational invariance is hardly advantageous.

\subsection{Solution method}

For a detailed description of the solution method, we refer to [7]. Here we give a brief summary.

For the nonlinear system of first-order discretized equations, symmetric point Gauss-Seidel relaxation is used. In here, one or more (exact) Newton steps are used for the collective updates of the four state vector components in each finite volume. Nonlinear multigrid is applied as an acceleration technique, the process is started by nested iteration.

For the higher-order accurate operator the same method leads to poor convergence or even divergence. As a remedy, we use iterative defect correction as an outer iteration for nonlinear multigrid applied, again, to the first-order discretized equations.

The application of the (exact) Newton method requires the convective and diffusive fluxes to be continuously differentiable (The diffusive fluxes as described in the previous section already fulfil this requirement.)

\section{DISCRETIZATION OF CONVECTIVE PART}

\subsection{Approximate Riemann solver}

As approximate Riemann solver for the Euler equations, we preferred Osher's scheme [12]. Reasons for this preference were: (i) its continuous differentiability, and (ii) its consistent treatment of boundary conditions. Here, the question arises whether it is still a good choice to use Osher's scheme when typical Navier-Stokes features such as shear, separation and heat conduction also have to be resolved. We shouid make a choice again.

Since continuous differentiability is an absolute requirement for the success of our solution method, and since we know no other approximate Riemann solvers with this property than Osher's and van Leer's [9], our choice is confined to these two only. So far, van Leer's scheme is more widespread in the field of Navier-Stokes than Osher's scheme [13, 14, 18]. Probably, the main reason for this is its greater conceptual and operational simplicity appealing from its first publications. However, recent publications on Osher's scheme, such as [6, 16], may help to reduce this difference.

With as next requirement the accurate modelling of physical diffusion, in fact, the definite choice can be made already. In [9], van Leer stated already that his flux vector splitter cannot preserve a steady contact discontinuity. Since a discrete shear layer may be interpreted as a layer of contact discontinuities, doubt rose already about the suitability of van Leer's scheme for Navier-Stokes codes. Recently, this doubt was confirmed in [11] where van Leer et al made a qualitative analysis (supplemented with numerical experiments) for various upwind schemes. There, Osher's scheme turned out to be better than van Leer's scheme, in particular for the resolution of boundary layer flows.

To shed some light on how large this difference in quality is, here results of a quantitative error analysis are presented for both Osher's and van Leer's scheme. The analysis is confined to the steady, 2D, isentropic Euler equations for a perfect gas with $\gamma=1$ :

$$
\frac{\partial f(q)}{\partial x}+\frac{\partial g(q)}{\partial y}=0
$$

with

$$
f(q)=\left(\begin{array}{l}
\rho u \\
\rho\left(u^{2}+c^{2}\right) \\
\rho u v
\end{array}\right), g(q)=\left(\begin{array}{l}
\rho v \\
\rho v u \\
\rho\left(v^{2}+c^{2}\right)
\end{array}\right),
$$

where $c$ is constant. (The choice of $2 \mathrm{D}$ equations allows us to consider a boundary layer flow in the analysis.) In [8], for both upwind schemes, the system of modified equations is derived, considering (i) a first-order accurate, square finite volume discretization, and (ii) a subsonic flow with $u$ and $v$ positive, and $\rho \approx$ constant. Neglecting the density variation, the systems of modified equations are, for Osher: 


$$
\frac{\partial f(q)}{\partial x}+\frac{\partial g(q)}{\partial y}-h \frac{\rho}{2 c}\left[\frac{\partial}{\partial x}\left[\begin{array}{l}
\frac{1}{2} \frac{\partial\left(u^{2}\right)}{\partial x} \\
\left(u^{2}+c^{2}\right) \frac{\partial u}{\partial x} \\
u v \frac{\partial u}{\partial x}+u c \frac{\partial v}{\partial x}
\end{array}\right]+\frac{\partial}{\partial y}\left[\begin{array}{l}
\frac{1}{2} \frac{\partial\left(v^{2}\right)}{\partial y} \\
v u \frac{\partial v}{\partial y}+v c \frac{\partial u}{\partial y} \\
\left(v^{2}+c^{2}\right) \frac{\partial v}{\partial y}
\end{array}\right)\right]=O\left(h^{2}\right),
$$

and for van Leer:

$$
\frac{\partial f(q)}{\partial x}+\frac{\partial g(q)}{\partial y}-h \frac{\rho}{2 c}\left[\frac{\partial}{\partial x}\left[\begin{array}{l}
\frac{1}{2} \frac{\partial\left(u^{2}\right)}{\partial x} \\
2 c^{2} \frac{\partial u}{\partial x} \\
\frac{1}{2} \frac{\partial\left(\left(u^{2}+c^{2}\right) v\right)}{\partial x}
\end{array}\right]+\frac{\partial}{\partial y}\left[\begin{array}{l}
\frac{1}{2} \frac{\partial\left(v^{2}\right)}{\partial y} \\
\frac{1}{2} \frac{\partial\left(\left(v^{2}+c^{2}\right) u\right)}{\partial y} \\
2 c^{2} \frac{\partial v}{\partial y}
\end{array}\right]\right) O\left(h^{2}\right)
$$

In both first-order error terms, a typical Navier-Stokes flow solution is substituted, which clearly shows the differences between both schemes. As flow, we consider an incompressible semi-infinite flat plate flow. For simplicity, for this we do not use the exact Blasius solution, but Lamb's approximate solution which reads

$$
\left[\begin{array}{l}
\rho \\
u \\
v
\end{array}\right]=\left[\begin{array}{l}
P \\
U \sin \left(\frac{\pi}{2} \frac{\sqrt{\operatorname{Re} / x}}{5} y\right) \\
\frac{y}{2 x} u(x, y)+\frac{5}{\pi} \frac{U}{\sqrt{\operatorname{Rex}}}\left\{\cos \left(\frac{\pi}{2} \frac{\sqrt{\operatorname{Re} / x}}{5} y\right)-1\right\}
\end{array}\right),
$$

with $P$ and $U$ constant. Substituting the solution vector (2.5) into the error vector of both (2.3) and (2.4), considering the boundary layer edge

$$
y=\delta(x) \equiv \frac{5}{\sqrt{\operatorname{Re} / x}}
$$

at $x=1$, and taking the ratio of absolute values of both error vectors, using $\operatorname{Re} \gg 1$ (which is our interest), we find

$$
\frac{\text { error Osher }}{\text { error van Leer }}=\left(\begin{array}{c}
\left.1-\frac{2}{\pi}\right) \frac{5}{\sqrt{R e}} \frac{U}{C} \\
1 / 2
\end{array}\right) \text {, }
$$

where we write $c=C$. From (2.7) it appears that van Leer's scheme deteriorates compared to Osher's scheme for increasing $R e$. Assuming the reliability of (2.7) for rather small $R e$, it appears that already for $R e>\{5(1-2 / \pi) U / C\}^{2}$, where $U / C<1$, Osher's scheme definitely is to be preferred above van Leer's scheme.

To ensure a continuous transition along a solid wall boundary from the Navier-Stokes flow regime to the Euler flow regime, for van Leer's scheme it will be necessary to impose only the Euler boundary condition to the convective part. So, for a non-permeable solid wall this means that one should only impose a zero normal velocity component to the convective part (though all boundary conditions to the diffusive part, i.e. a zero normal and tangential velocity component, and some temperature condition). By not imposing the no-slip and temperature boundary condition to the convective part, we avoid that it 'feels' the severe contact discontinuity in the realistic case of a boundary layer flow on a very coarse grid and an outer flow with $M$ not small. Such a contact discontinuity will be erroneously spread by van Leer's scheme, and cause that there is some finite, rather low value of $R e$ above which the solution is insensitive to $R e$-variation.

Osher's scheme can preserve a steady contact discontinuity as long as it is aligned with the grid. Application of (commonly used) body-fitted grids guarantees this alignment along solid walls. Therefore, with a body-fitted grid, Osher's scheme does not need the careful solid wall - boundary condition treatment as proposed for van Leer's scheme. 


\subsection{Higher-order accuracy}

As mentioned in section 1.2.1, higher-order accuracy is obtained by applying low-degree piecewise polynomial functions through two or three adjacent volume states. The polynomials are given by van Leer's $\kappa$-scheme [10]

$$
\begin{aligned}
& q_{i+1 / 2, j}^{l}=q_{i, j}+\frac{1+\kappa}{4}\left(q_{i+1, j}-q_{i, j}\right)+\frac{1-\kappa}{4}\left(q_{i, j}-q_{i-1, j}\right), \\
& q_{i+1 / 2, j}^{r}=q_{i+1, j}+\frac{1+\kappa}{4}\left(q_{i, j}-q_{i+1, j}\right)+\frac{1-\kappa}{4}\left(q_{i+1, j}-q_{i+2, j}\right),
\end{aligned}
$$

with $\kappa \in[-1,1]$. For $\kappa=-1,0,1$, we have the fully one-sided upwind, the Fromm and the central scheme, respectively.

The aim now is to optimize $k$. For this purpose, we consider the scalar model equation

$$
\frac{\partial u}{\partial x}+\frac{\partial u}{\partial y}-\epsilon\left(\frac{\partial^{2} u}{\partial x^{2}}+\frac{\partial^{2} u}{\partial x \partial y}+\frac{\partial^{2} u}{\partial y^{2}}\right)=0 .
$$

On a square grid, a finite volume discretization which uses the $\kappa$-scheme for convection and the central scheme for diffusion, yields as modified equation

$$
\begin{aligned}
& \frac{\partial u}{\partial x}+\frac{\partial u}{\partial y}-\epsilon\left(\frac{\partial^{2} u}{\partial x^{2}}+\frac{\partial^{2} u}{\partial x \partial y}+\frac{\partial^{2} u}{\partial y^{2}}\right)+ \\
& +h^{2}\left\{\frac{\kappa-1 / 3}{4}\left(\frac{\partial^{3} u}{\partial x^{3}}+\frac{\partial^{3} u}{\partial y^{3}}\right)-\frac{\epsilon}{12}\left(\frac{\partial^{4} u}{\partial x^{4}}+2 \frac{\partial^{4} u}{\partial x^{3} \partial y}+2 \frac{\partial^{4} u}{\partial x \partial y^{3}}+\frac{\partial^{4} u}{\partial y^{4}}\right)\right\}=O\left(h^{3}\right) .
\end{aligned}
$$

As optimal value for $\kappa$, we define: the value that gives the highest possible accuracy, i.e. third-order accuracy in this case. Assuming the reliability of the underlying Taylor series expansion, from (2.10), we find for this value

$$
\kappa=\frac{1}{3}\left\{1+\epsilon\left(\frac{\partial^{4} u}{\partial x^{4}}+2 \frac{\partial^{4} u}{\partial x^{3} \partial y}+2 \frac{\partial^{4} u}{\partial x \partial y^{3}}+\frac{\partial^{4} u}{\partial y^{4}}\right) /\left(\frac{\partial^{3} u}{\partial x^{3}}+\frac{\partial^{3} u}{\partial y^{3}}\right)\right\} .
$$

Since convection dominated problems, problems with $\epsilon \ll 1$, are our interest, we assume the above diffusion-dependence of $\kappa$ to be negligible, which simply leads to $\kappa=1 / 3$.

\subsection{Monotonicity}

To preserve monotonicity, we construct a limiter which is based on the $\kappa=1 / 3$-scheme. For this, we use the monotonicity theory of Spekreijse [15], an extension of Sweby's theory [17], allowing more freedom in the limiter construction.

For the limited, higher-order, left and right state components, we write

$$
\begin{aligned}
& q_{i+1 / 2, j}^{l(k)}=q_{i, j}^{(k)}+\frac{1}{2} \phi\left(R_{i, j}^{(k)}\right)\left(q_{i, j}^{(k)}-q_{i-1, j}^{(k)}\right), \\
& q_{i+1 / 2, j}^{r(k)}=q_{i+1, j}^{(k)}+\frac{1}{2} \phi\left(1 / R_{i+1, j}^{(k)}\right)\left(q_{i+1, j}^{(k)}-q_{i+2, j}^{(k)}\right),
\end{aligned}
$$

with $k=1,2,3,4, \phi(R)$ the limiter, and

$$
R_{i, j}^{(k)}=\frac{q_{i+1, j}^{(k)}-q_{i, j}^{(k)}}{q_{i, j}^{(k)}-q_{i}^{(k)}-1, j}
$$

The limited $\kappa=1 / 3$-scheme can be written in the one-sided form $(2.12 \mathrm{a}-\mathrm{b})$ as

$$
\begin{aligned}
& q_{i+1 / 2, j}^{l(k)}=q_{i, j}^{(k)}+\frac{1}{2} \xi\left(R_{i, j}^{(k)}\right)\left(\frac{1}{3}+\frac{2}{3} R_{i, j}^{(k)}\right)\left(q_{i, j}^{(k)}-q_{i}^{(k)}-1, j\right), \\
& q_{i+1 /, j}^{r(k)}=q_{i+1, j}^{(k)}+\frac{1}{2} \xi\left(1 / R_{i+1, j}^{(k)}\right)\left(\frac{1}{3}+\frac{2}{3} / R_{i+1, j}^{(k)}\right)\left(q_{i+1, j}^{(k)}-q_{i+2, j}^{(k)}\right) .
\end{aligned}
$$

Notice that for $\xi(R)=1$ we have the (non-limited) $\kappa=1 / 3$-scheme, and that $\xi(R)$ defines the limiter $\phi(R)$ by

$$
\phi(R)=\xi(R)\left(\frac{1}{3}+\frac{2}{3} R\right) .
$$


General requirements to be fulfilled by $\xi(R)$ are: $\xi(1)=1$ to preserve higher-order accuracy, and: $\xi(0)=0$ and boundedness for large $|R|$ to preserve monotonicity. For the latter, we require that $\lim _{R \rightarrow \pm \infty} \xi(R)\left(\frac{1}{3}+\frac{2}{3} R\right)=1$. To make the limiter now a $k=1 / 3$-limiter, we require that $\xi^{\prime}(1)=0$. (This last requirement makes the limiter tangential to the $\kappa=1 / 3$-scheme in the monotonicity region [15].) Imposing these five requirements to the general form

$$
\xi(R)=\frac{\alpha_{1} R^{2}+\alpha_{2} R+\alpha_{3}}{\alpha_{4} R^{2}+\alpha_{5} R+1},
$$

we find with (2.15)

$$
\phi(R)=\frac{2 R^{2}+R}{2 R^{2}-R+2} .
$$

\section{NUMERICAL RESULTS}

\subsection{Flow problems}

To evaluate the discretization method, the following flow problems are considered: (i) a subsonic flat plate flow with $M=0.5$ and $R e$ ranging from $10^{2}$ up to $10^{100}$, and (ii) a supersonic flat plate flow with oblique shock wave - boundary layer interaction at $M=2, R e=2.9610^{5}$. The latter problem stems from [3].

For the subsonic flow problem, the Blasius solution is used as a reference. The grids used for this flow problem are all composed of square finite volumes. As coarsest grid in all multigrid computations, we use a $4 \times 2$-grid.

For the supersonic flow problem, the experimental results from [3] are used as a reference. Here, in all multigrid computations a $5 \times 2$-grid is applied as coarsest grid. The grid was optimized for convection by introducing a stretching in flow direction, and in particular by aligning it with the impinging shock wave. A grid adaptation for diffusion was realized by introducing a stretching in crossflow direction.

For both flow problems, we use $\gamma=1.4$ and $\operatorname{Pr}=0.71$. For further details about the implementation of both problems, we refer to [8].

\subsection{Osher versus van Leer}

To show at first the benefit of the solid wall - boundary condition treatment as proposed for van Leer's scheme in section 2.1, we consider the subsonic flat plate flow at $R e=10^{100}$. For both Osher's and van Leer's scheme we compute the flow on a $64 \times 32$-grid, using the first-order accurate discretization and imposing to the convective part, successively: (i) non-permeability, no-slip and no-heattransfer, and - carefully - (ii) non-permeability only. The numerical results obtained are given in fig. 3.1. For the case with all Navier-Stokes boundary conditions imposed, it appears that van Leer's scheme severely thickens the thin layer, whereas Osher's scheme preserves it. With the careful approach, both schemes preserve the layer.

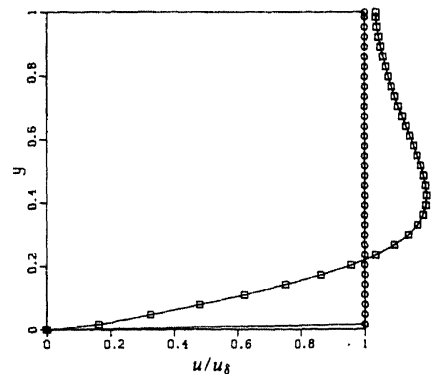

a. $u=v=0, \frac{\partial c}{\partial y}=0$.

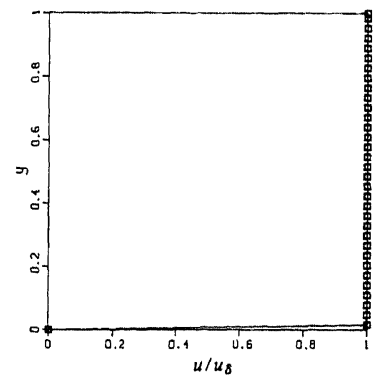

b. $\quad v=0$ only. Fig. 3.1. Velocity profiles at $x=0$ for the subsonic flat plate flow at $R e=10^{100}$ and $h=\frac{1}{32}$,
for two solid wall - boundary condition treatments (O: Osher, $\square$ : van Leer). 
Using the careful boundary condition treatment, for both schemes we perform an experiment with $h$ - and $R e$-variation, using again the first-order accurate discretization. Numerical results obtained are given in fig. 3.2. The results show the superiority of Osher's scheme, in particular for high mesh Reynolds numbers. The deterioration of van Leer's scheme with respect to Osher's scheme which occurs in fig. $3.2 \mathrm{~b}$ for increasing $R e$, is in agreement with the analytical results presented in section 2.1.

All numerical results presented hereafter were obtained with Osher's scheme only.
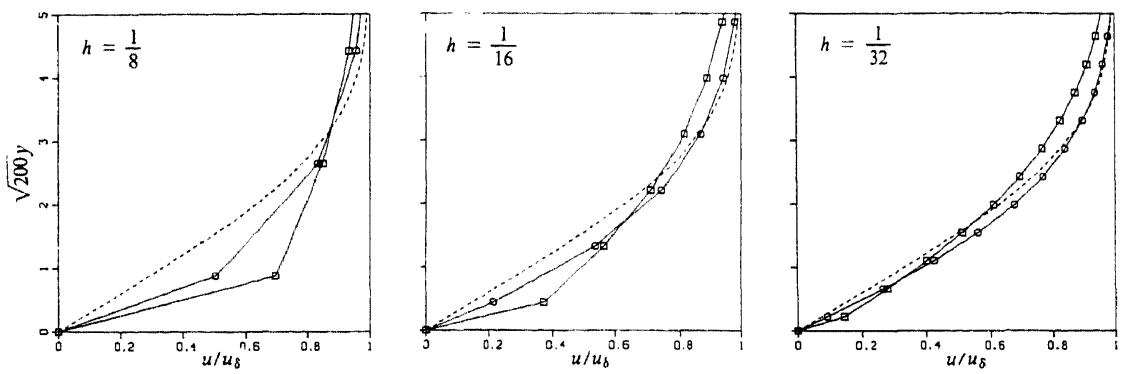

a. $h$-variation with $R e=100$ (O : Osher, $\square$ : van Leer).
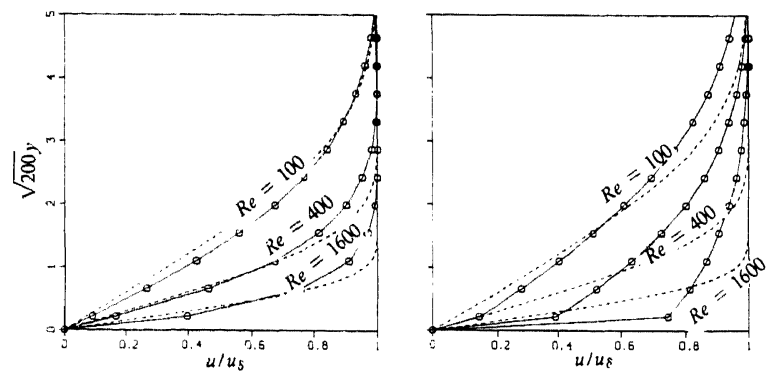

b. Re-variation with $h=1 / 32$ (left: Osher, right: van Leer).

Fig. 3.2. Velocity profiles at $x=0$ for the subsonic flat plate flow (-----: Blasius solution).

\subsection{Monotone higher-order accuracy}

To evaluate our monotone higher-order accurate scheme, we consider the supersonic flat plate flow. At first, we evaluate monotonicity, and next higher-order accuracy.

For monotonicity, we compute the Euler flow solution on the $80 \times 32$-grid given in fig. 3.3a, using the $k=1 / 3$-scheme with and without limiter. Numerical results obtained are given in fig. 3.3b. The results clearly show that the limiter does what it is supposed to do: making the solution monotone.

For higher-order accuracy, we compute on the same grid the Navier-Stokes solution, using now the limited $\kappa=1 / 3$-scheme and the first-order scheme. A comparison is made with the experimental results from [3]. The results, given in fig. 3.3c, clearly show the need for higher-order accuracy. The first-order accurate surface pressure distribution lacks the plateau in the pressure distribution, which indicates that its solution has no separation bubble (i.e. no separation and no re-attachment). In agreement with the experimental results, the limited higher-order accurate surface pressure distribution does have a separation bubble. The quantitative differences still existing between the limited higher-order and measured surface pressure distribution must be due to uncertain influences in both the experiment and the computation. (As far as the experiment is concerned, this might be crossflow influences, non-observed though influential turbulence, some slight heat transfer through the wall, and so on. Concerning the computation, this might be for instance the neglect of temperature dependence in the diffusion coefficients.) 


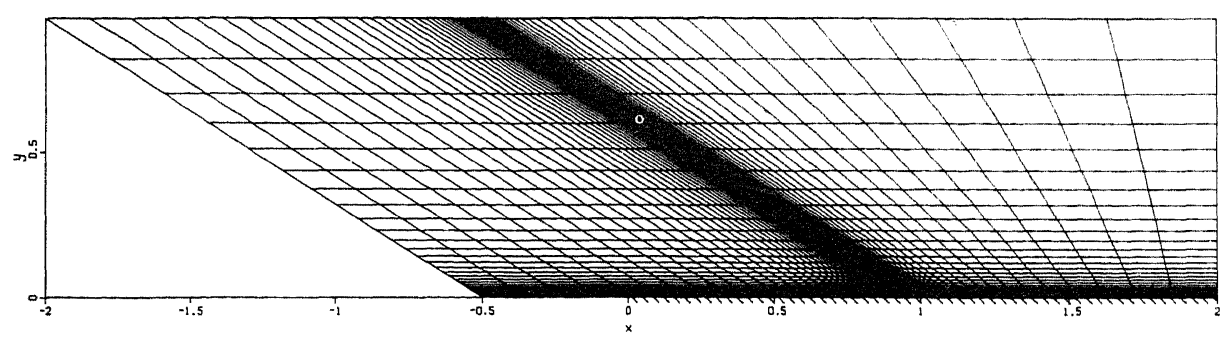

a. Finest grid applied $(80 \times 32)$.

b. Inviscid surface pressure distributions ( $\bigcirc:$ limited $\kappa=\frac{1}{3}, \quad \square:$ non-limited $\kappa=\frac{1}{3}$ ).

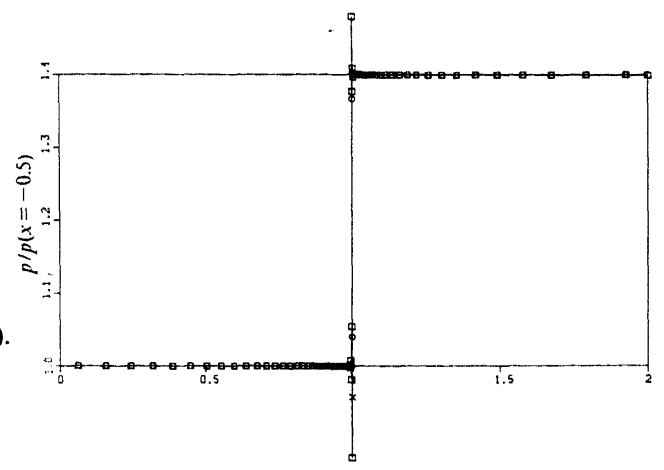

c. Viscous surface pressure distributions ( $O$ : limited $\kappa=\frac{1}{3}, \Delta:$ first-order, : : measured).

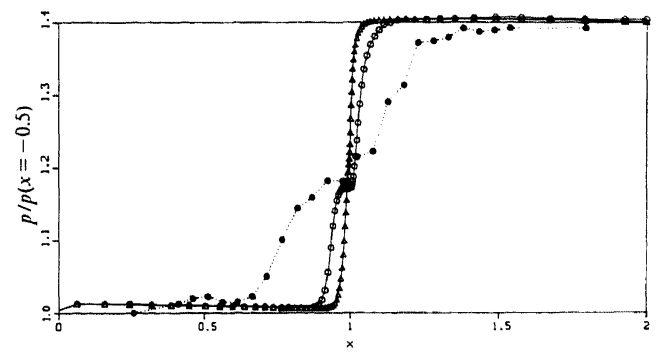

Fig. 3.3. Results supersonic flat plate flow on oblique grid.

\subsection{False diffusion}

It should be noticed that by presenting for the supersonic flat plate flow, besides the viscous solution (obtained with the limited $\kappa=1 / 3$-scheme) also the corresponding inviscid solution, insight was given about the amount of false diffusion present in the viscous solution. The fact that the present method is hybrid in the sense that it can be used for both Navier-Stokes and Euler flows makes it easy to do this investigation. Omitting this investigation for the supersonic flat plate flow, when applying a commonly used rectangular grid, such as for instance the $80 \times 32$-grid shown in fig. 3.4a, leads to a viscous surface pressure distribution which seems to be very close to the experimental data (fig. 3.4b). However, the corresponding inviscid distribution indicates that this good resemblance is absolutely fake (fig. 3.4c). 
a. Finest grid applied $(80 \times 32)$

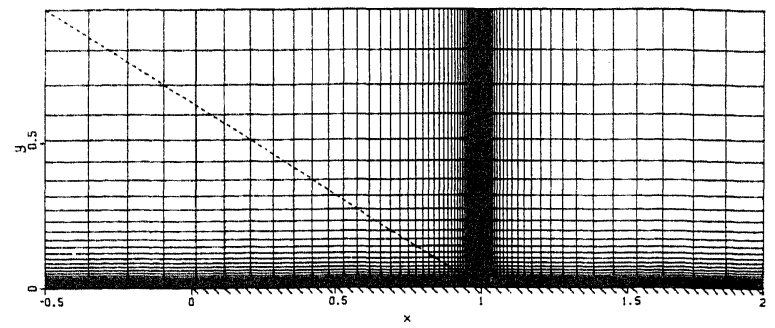

b. Viscous surface pressure distributions ( $O:$ limited $\kappa=\frac{1}{3}, \quad$ : measured).

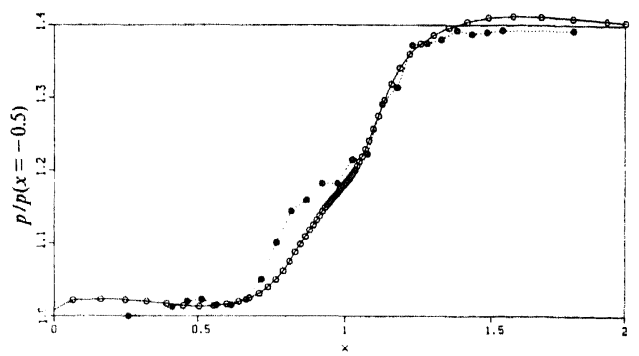

c. Inviscid surface pressure distrihutions, ( $\bigcirc:$ limited $\kappa=\frac{1}{3}$, $\bullet:$ measured).

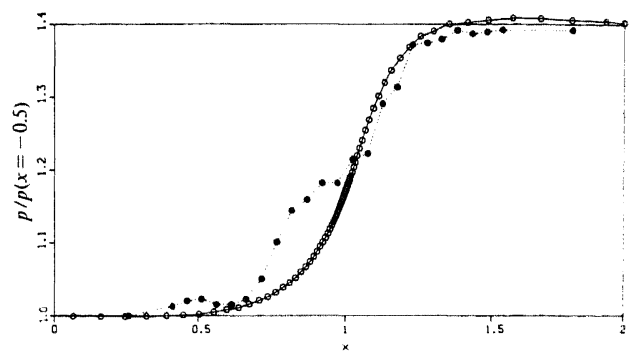

Fig. 3.4. Results supersonic flat plate flow on rectangular grid.

\section{CONCLUSIONS}

Theory and practice show that Osher's scheme leads to a more accurate resolution of boundary layers than van Leer's scheme. The difference in accuracy becomes larger with increasing Reynolds number. Already for rather low Reynolds numbers, the difference is such that, for engineering purposes, Osher's scheme is to be preferred above van Leer's scheme. An accidental circumstance is that Osher's scheme needs no special care in the application of solid wall - boundary conditions, whereas van Leer's scheme does. (To avoid, when still using van Leer's scheme, that there is some rather low Reynolds number above which the flow solutions are insensitive to Reynolds-variation, only the Euler boundary condition should be imposed to the convective part.)

It is important to investigate the reliability of any computed Navier-Stokes solution with respect to the numerical errors in the discretization of the convective part. The present code allows an easy check of false diffusion: the same code can be used for both viscous $(1 / \operatorname{Re}>0)$ and inviscid $(1 / R e=0)$ flow computations.

The discretization method is parameter-free; it needs no tuning. 


\section{REFERENCES}

1. S.R. Chakravarthy, K.Y. Szema, U.C. Goldberg, J.J. Gorski and S. Osher (1985). Application of a New Class of High Accuracy TVD Schemes to the Navier-Stokes Equations. AIAA-paper $85-0165$.

2. S.K. Godunov (1959). Finite Difference Method for Numerical Computation of Discontinuous Solutions of the Equations of Fluid Dynamics (in Russian, also Cornell Aeronautical Lab. Transl.). Math. Sbornik 47, 272-306.

3. R.J. Hakkinen, I. Greber, L. Trilling and S.S. Abarbanel (1958). The Interaction of an Oblique Shock Wave with a Laminar Boundary Layer. NASA-memorandum 2-18-59 W.

4. P.W. Hemker (1986). Defect Correction and Higher Order Schemes for the Multi Grid Solution of the Steady Euler Equations. Proceedings of the 2nd European Conference on Multigrid Methods (W. Hackbusch and U. Trottenberg, eds.), Cologne, 1985. Springer, Berlin.

5. P.W. Hemker and B. Koren (1988). Defect Correction and Nonlinear Multigrid for the Steady Euler Equations. Lecture Series on Computational Fluid Dynamics, von Karman Institute for Fluid Dynamics, Rhode-Saint-Genèse.

6. P.W. HeMker and S.P. SPEKREuSE (1986). Multiple Grid and Osher's Scheme for the Efficient Solution of the Steady Euler Equations. Appl. Num. Math. 2, 475-493.

7. B. KOREN (1988). Multigrid and Defect Correction for the Steady Navier-Stokes Equations. Proceedings of the 4th GAMM-Seminar Kiel on Robust Multi-Grid Methods, (W. Hackbusch, ed.), Kiel, 1988. Vieweg, Braunschweig (to appear).

8. B. Koren (1988). Upwind Discretization for the Steady Navier-Stokes Equations. Report NMR88xx, Centre for Mathematics and Computer Science, Amsterdam (to appear).

9. B. VAn Leer (1982). Flux-Vector Splitting for the Euler Equations. Proceedings of the 8th International Conference on Numerical Methods in Fluid Dynamics (E. Krause, ed.), Aachen, 1988. Springer, Berlin.

10. B. VAN Leer (1985). Upwind-Difference Methods for Aerodynamic Problems governed by the Euler Equations. Proceedings of the 15th AMS-SIAM Summer Seminar on Applied Mathematics (B.E. Engquist, S. Osher and R.C.J. Somerville, eds.), Scripps Institution of Oceanography, 1983. AMS, Rhode Island.

11. B. van Leer, J.L. Thomas, P.L. Roe and R.W. Newsome (1987). A Comparison of Numerical Flux Formulas for the Euler and Navier-Stokes Equations. AIAA-paper 87-1104.

12. S. OSHER and F. SOLOMON (1982). Upwind-Difference Schemes for Hyperbolic Systems of Conservation Laws. Math. Comp. 38, 339-374.

13. W. SCHRÖDER and D. HÄNEL (1987). An Unfactored Implicit Scheme with Multigrid Acceleration for the Solution of the Navier-Stokes Equations. Computers and Fluids 15, 313-336.

14. G. Shaw and P. Wesseling (1986). Multigrid Solution of the Compressible Navier-Stokes Equations on a Vector Computer. Proceedings of the 10th International Conference on Numerical Methods in Fluid Dynamics (F.G. Zhuang and Y.L. Zhu, eds.), Beijing, 1986. Springer, Berlin.

15. S.P. SPEKREIJSE (1987). Multigrid Solution of Monotone Second-Order Discretizations of Hyperbolic Conservation Laws. Math. Comp. 49, 135-155.

16. S.P. SpeKreISSE (1987). Multigrid Solution of the Steady Euler Equations. Ph.D.-thesis, Centre for Mathematics and Computer Science, Amsterdam.

17. P.K. SWEBY (1984). High Resolution Schemes using Flux Limiters for Hyperbolic Conservation Laws. SIAM J. Num. Anal. 21, 995-1011.

18. J.L. Thomas and R.W. Walters (1985). Upwind Relaxation Algorithms for the Navier-Stokes Equations. AIAA-paper 86-1501. 